ORIGINAL ARTICLE

\title{
Defining and classifying medical error: lessons for patient safety reporting systems
}

\author{
M Tamuz, E J Thomas, K E Franchois
}

See editorial commentary, p 8

Qual Saf Health Care 2004;13:13-20. doi: 10.1136/qshc.2002.003376

See end of article for authors' affiliations

.....................

Correspondence to:

Dr M Tamuz, University of Tennessee Health Science Center, Center for Health Services Research, 66

North Pauline, Suite 463, Memphis, TN 38163,

USA; mtamuz@utmem.edu

Accepted 19 October 2003
Background: It is important for healthcare providers to report safety related events, but little attention has been paid to how the definition and classification of events affects a hospital's ability to learn from its experience.

Objectives: To examine how the definition and classification of safety related events influences key organizational routines for gathering information, allocating incentives, and analyzing event reporting data.

Methods: In semi-structured interviews, professional staff and administrators in a tertiary care teaching hospital and its pharmacy were asked to describe the existing programs designed to monitor medication safety, including the reporting systems. With a focus primarily on the pharmacy staff, interviews were audio recorded, transcribed, and analyzed using qualitative research methods.

Results: Eighty six interviews were conducted, including 36 in the hospital pharmacy. Examples are presented which show that: (1) the definition of an event could lead to under-reporting; (2) the classification of a medication error into alternative categories can influence the perceived incentives and disincentives for incident reporting; (3) event classification can enhance or impede organizational routines for data analysis and learning; and (4) routines that promote organizational learning within the pharmacy can reduce the flow of medication error data to the hospital.

Discussion: These findings from one hospital raise important practical and research questions about how reporting systems are influenced by the definition and classification of safety related events. By understanding more clearly how hospitals define and classify their experience, we may improve our capacity to learn and ultimately improve patient safety.
$\mathrm{P}$ atient safety experts debate how to define and classify events such as errors, near misses, and adverse events that should be monitored by patient safety reporting systems, ${ }^{12}$ but relatively little attention has been paid to how this process actually occurs in healthcare organizations. Even less is known about how definitions and classification schemes affect an organization's ability to investigate and draw conclusions from its experience.

We propose that definitions and classification schemes can have profound implications for a hospital's capacity to gather information about and learn from events related to patient safety. Clearly, reaching agreement on definitions and classification schemes can improve the reliability of classification. However, our main argument is that the choice of definitions and classification schemes is critical because of the influence of event categorization on the processes used by organizations in gathering and making sense of information. We base our argument on concepts of categorization originating in cognitive psychology ${ }^{34}$ and developed in organization theory. ${ }^{56}$

We also draw upon empirical research on aviation safety reporting systems indicating that the categorization of events into one category or another serves as a toggle switch that activates a choice of organizational routines. ${ }^{7}$ In air traffic control, for example, if a near miss did not technically fit the definition of an "operational error", it was not reported to or included in the error database. ${ }^{8}$ This practice made sense for individual air traffic controllers who should not be held accountable for close calls that occurred when the aircraft was solely under the control of the pilot, but air traffic system regulators can lose valuable information about hazardous conditions when this definition is applied. We recognize important differences between the fields of aviation and health care ${ }^{9}$ and their respective safety monitoring systems, but we believe that research on aviation safety reporting systems can provide directions for research on hospital reporting systems. ${ }^{10} 11$

In this paper we describe how the definition and classification of safety related events influences key organizational routines for gathering information, allocating incentives, and analyzing data in hospitals (fig 1). We illustrate these processes with examples selected from our interviews with pharmacy staff in a tertiary care teaching hospital. We also suggest questions for researchers and hospital administrators to consider as they seek to improve patient safety.

\section{METHODS}

The results from our preliminary analysis of interviews from one hospital pharmacy are presented, with supplemental data drawn from interviews with nurses, physicians, and administrators. To augment the interviews we also took extensive field notes, collected documents (such as reporting forms), and observed routine activities. Drawing mainly from interviews with hospital pharmacy staff, we developed research and practical questions. This study is part of an ongoing research project in which we examine how two hospitals and their pharmacies seek to learn how to improve medication safety.

\section{Organizational setting}

The study was conducted in a tertiary care teaching hospital which was chosen because it had (1) a high volume of 


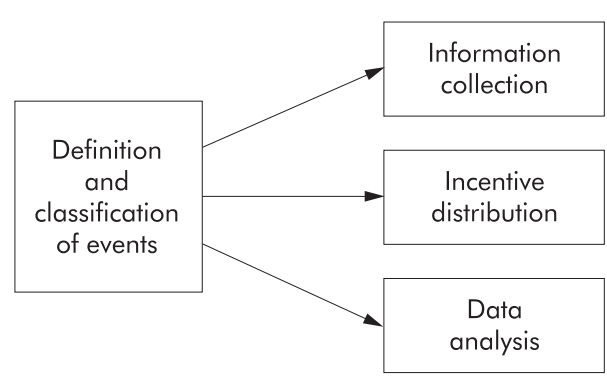

Figure 1 Conceptual framework.

medication usage, (2) high potential for adverse drug events (due to a vulnerable patient population and/or the use of hazardous medications), and (3) existing medication safety programs including reporting systems. We concentrated on medication errors because they are one of the most frequent types of error in hospitals, ${ }^{12}$ and we focused our inquiry on the pharmacy so we could explore these issues in depth. Detailed analyses of how other professionals classify errors will be the subject of additional studies.

\section{Study participants}

The sample was designed to reflect the perspectives of participants working at different levels in the organizational hierarchy and representing diverse professional groups. Study participants were chosen from positions ranging from policy makers such as vice presidents to front line employees such as pharmacy technicians. We randomly selected healthcare practitioners from the pharmacy and patient care unit and chose administrators in key positions such as those engaged in quality assurance and risk management. We also added study participants in relevant positions who were identified during the course of the study, such as practitioners involved in various quality improvement committees. Patients were not interviewed. Although this study focuses primarily on the pharmacy, we also took into account the perspectives of other professionals.

\section{Interviews}

A semi-structured interview protocol was developed to guide our interviews. Participants were asked to describe and "walk us through" the existing programs designed to monitor medication safety, and specific open ended questions about the patient safety reporting systems were posed. By asking descriptive questions we elicited the participants' knowledge of how the reporting systems operated as well as their perceptions of it. By posing indirect questions and asking participants to provide examples, we sought to obtain answers that did not reflect socially expected responses. Following accepted qualitative research techniques, ${ }^{13}$ the interview protocol was revised and refined as the study progressed.

Given the sensitive topic of the interview, we emphasized that the interviews were voluntary, confidential, and being conducted solely for research purposes. With regard to informed consent, subjects were told that the study goal was to learn "how the hospital monitors the safety of the ordering, dispensing and distribution of medications". To encourage participants to speak freely, we emphasized that (1) we were from a university (not the hospital); (2) no names would be used and the hospital also would not be identified; (3) no one in the hospital could listen to the tapes or read the transcripts; and (4) most participants were chosen by chance. We interviewed study participants in the hospital during regular working hours in a private location outside the pharmacy or patient care unit.

The interviews were tape recorded with the permission of the participants. The recordings were transcribed and any transcription errors were corrected, and the audio tapes were retained to verify the tone and meaning of expressions.

\section{Analysis of data}

Examples were chosen from the interviews by combining a detailed reading of the field notes with an automated search for key words. Using the field notes as a guide, we chose several interviews in which participants discussed definitions of safety related events. These interviews were analyzed to derive a list of key words that could be used to find additional passages related to definitions. Applying a key word search, HyperResearch 2.03 was used to code the additional interview transcripts for definitions of safety related events.

The research team members, including the investigators and research assistants, chose examples by a sequential review of the interview excerpts produced by the computerized search. Firstly, we discarded excerpts that included key words but did not relate to the topic. Secondly, each team member reviewed the coded excerpts and selected those that best illustrated the processes of event definition. Thirdly, we conducted a sceptical examination of our data analyses and interpretations by actively looking for counter examples to contradict the selected interview passages. Finally, by consensus, the investigators chose examples to illustrate the definition and classification of safety related events.

We also identified themes in the description of organizational processes that emerged from the interviews. Our analysis was guided, in part, by examining the pharmacy interviews in the context of aviation research. ${ }^{7}$ We also inferred key organizational processes such as methods of categorization and communication channels from our analysis of the selected excerpts described above. For example, we investigated methods of categorization by tracing the steps that the organization followed after an event was first defined. In an iterative process we revised and refined the themes that we observed in the organizational routines as new examples were added. ${ }^{13}$

The study was approved both by the Institutional Review Board designated by the hospital and the Committee for the Protection of Human Subjects at the University of Texas Health Science Center. We conducted 76 interviews during a 3 month period at the end of 2001 and completed the remaining 10 follow up interviews within 1 year of the first interview.

\section{RESULTS}

Eighty six confidential interviews were conducted with a random sample of 36 pharmacy staff members, 36 members of a patient care unit (including nurses and physicians), and a selected sample of 14 key hospital administrators. Four people declined to participate, approximately one from each group. The interviews ranged in length from 30 minutes to about 12 hours. All three investigators took part in some of the interviews, with $85 \%$ of the interviews conducted by two investigators (MT and KF).

In general, the participants were remarkably open and forthcoming. The field notes recorded by the investigators included their assessment of the participants' candour. At one extreme, a few nurses treated the interview like a legal deposition, giving brief answers, refraining from providing examples, and claiming memory losses. More often pharmacists, nurses, and physicians apparently used the interview to talk about events that concerned them. Participants often 
spontaneously described their first hand knowledge of a medication error or potentially harmful situation, regardless of the question asked by the interviewer.

\section{Definitions and information collection}

Definitions that hinder incident reporting to the hospital

When we asked the hospital pharmacy staff how they defined a reportable incident, we found that the classification of an error as a reportable incident depended on where the event was detected. As a pharmacy management team member explained:

"Really, the difference between an incident and an error, an incident is [when] it got outside our department". (Pharmacy management team 28)

If an error such as a wrong dose was detected while the medication was still under the control of the pharmacy, it was not defined as a reportable incident. However, if an error was detected when the drug had been sent outside the pharmacy, it could be considered a reportable incident and possibly be reported to the hospital incident reporting system. For example, a pharmacist referred to an error caught in the pharmacy as a "non-event". A member of the pharmacy management team underscored that pharmacists routinely double checked the work of those who wrote and filled orders and caught their mistakes, a term he used interchangeably with medication errors:

"A mistake is something that, to me, is just within our department. It's even because we are tailored to catch that. That's part of our process; so we accept that as part of the flow, unfortunately." (Pharmacy management team 28)

From the pharmacy's perspective, this categorization scheme makes sense. If a medication error was detected and corrected before the drugs left the pharmacy, then the error could not pose a danger to a patient. However, when a pharmacist corrected a seemingly routine error, that same type of error could potentially threaten future patients. The classification of errors as routine or as non-events resulted in a loss of information to the hospital because some of the errors detected in the pharmacy originated in part from actions taken outside the pharmacy.

\section{Definitions that promote learning in a hospital pharmacy}

Errors corrected within the pharmacy and categorized as "non-events" did not result in a complete loss of information and did not hamper the pharmacy's capacity to learn from its experience. We found three examples of learning that occurred in the pharmacy despite the loss of information to the hospital.

\section{Interventions}

The pharmacy used the term "interventions" to define and classify some of the events detected within the pharmacy. For example, if a pharmacist noticed a wrong dose during a

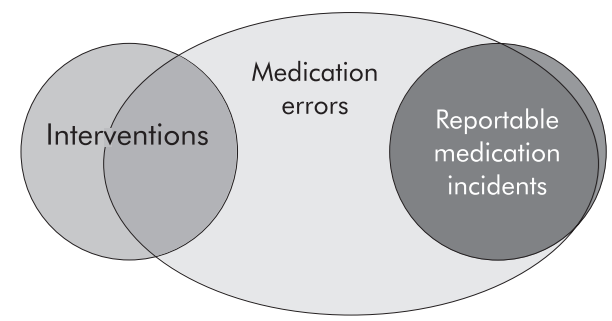

Figure 2 Classification of safety related medication events described by study participants. computer order entry, called the physician, and corrected the error before the medication left the pharmacy, it was defined as an intervention (fig 2). As a pharmacy management team member explained:

"Pharmacists' interventions are extremely important. These are the [telephone] calls that turn what we call prescribing errors into changed orders, and if they didn't call, then the prescribing errors would fall into the med incident reporting program." (Pharmacy management team 79)

Staff pharmacists were encouraged to keep track of and report the interventions they made, including the correction of physician prescribing errors, clarifications of prescriptions, and proactive recommendations by pharmacists such as alternative dosing methods. Seasoned pharmacy personnel reviewed the intervention data and looked for revealing trends. Thus, the pharmacy used information about some of the errors that were detected within the pharmacy but not reported to the hospital's incident reporting system.

\section{Informal definitions}

Pharmacists also generated informal definitions of safety related events. Some pharmacists used "good catch" to define the detection of an error by noticing subtle clues. Others discussed an "accident waiting to happen" or noticed a potentially dangerous situation before it resulted in an error, such as similarly named drugs placed in alphabetical order on a shelf. Yet another pharmacist described a potentially dangerous situation as:

"That's kinda setting me up for a problem." (Pharmacist 19)

These informally defined events were discussed among staff during breaks, in staff meetings, and via electronic mail, as a pharmacist recounted:

"If it's something that may happen for multiple patients, some people put an e-mail out about that 'This almost occurred, everybody be careful'. That kind of...pass it on through e-mail without specifying certain people. You know, 'Heads up! We made this mistake, somebody else might, so just watch, be careful'." (Pharmacist 9)

Staff pharmacists caught their own errors within the pharmacy and enabled their colleagues to learn from their experience.

\section{Organizational learning programs}

The pharmacy management team promoted informal and formal organizational learning programs or sets of related routines that actively gathered and used the information on potential problems identified by the staff pharmacists. Most of the pharmacists we interviewed described how they freely expressed their concerns to their supervisors regarding the errors that were caught within the pharmacy. As a pharmacist described:

"One of the pharmacists would come up with the idea and then kind of talk informally at different times to the other pharmacists, and they said, 'Oh, yeah! That's a great idea.' Then one of those pharmacists would go speak to her [a supervisor], also informally. Usually when she comes up on those morning rounds she'd stop by and we'd say, 'Hey, [supervisor's name], we had an idea. What do you think of getting rid of that [particular dosage]?'And she'd say: 'Oh, well, that's a good idea'." (Pharmacist 1)

Indeed, an informal learning system emerged within the pharmacy, supported and orchestrated by the pharmacy management. As a pharmacist said:

"But as far as our department's [the pharmacy] concerned, it's really an open-door policy. Just tell 'em what you're thinking, and they'll mull it over downstairs. I guess if they think it's good, then I guess the wheels get in motion. If they have some questions, they call you and they talk a little bit more about it." (Pharmacist 8)

In an informal learning program the management staff often responded to the concerns voiced by the pharmacists by 
conducting ad hoc experiments in which the pharmacists collected data to evaluate whether a particular "fix" actually corrected the problem. In a formal program, individual pharmacists presented a written proposal to the pharmacy director, conducted a study to test their proposed solutions, and presented their findings to the pharmacy staff.

These examples illustrate how the definition and categorization of events can influence organizational routines for gathering and analyzing data. Although errors that were caught and corrected within the pharmacy were not classified as reportable "incidents", the pharmacy and its staff developed alternative definitions ("interventions") and categorizations ("set ups") for some of these events and used information about them when deciding whether to change pharmacy procedures.

\section{Categorization and the allocation of incentives}

We also examined how the classification of a similar type of event could alternatively provide incentives or disincentives for reporting. For example, if a pharmacist detected a wrong dose, called a physician to correct it, and reported her intervention, then it would trigger a routine for the distribution of rewards and directly provide the pharmacist with an incentive to report future interventions. In contrast, if a nurse noticed the same mistaken dose outside the pharmacy, classified the error as a reportable incident, and submitted it to the hospital incident reporting system, then a very different set of routines would be set in motion. The pharmacy could hold the pharmacist involved accountable for his failure to catch the wrong dose, thus indirectly giving the pharmacist a disincentive for reporting other errors in which he or his colleagues were involved. We present examples from our interviews to describe how the distribution of (or reduction in) rewards based on the performance of pharmacists influenced their incentives for reporting interventions and incidents.

\section{Incentives for reporting}

Pharmacy managers rewarded the staff for making interventions, both formally in annual performance evaluations and informally through praise and recognition. As a key member of the pharmacy management team explained, the pharmacy managers tallied the number of interventions performed by pharmacists:

"We would hope they would record more [interventions] and that's part of our performance appraisal". (Pharmacy management team 79)

Indeed, several pharmacists mentioned that they routinely made interventions throughout the year, but reported them more diligently as the time for the annual performance evaluations approached. During the performance appraisal each pharmacist was assessed, partly based on the number of interventions he or she reported.

Supervisors commended pharmacists for making interventions, as a pharmacy manager noted:

"They're [the pharmacists] doing the right thing if they're making the interventions and the docs are changing the orders". (Pharmacy management team 79)

Occasionally, a pharmacy supervisor praised a pharmacist or pharmacy technician for making a "great catch":

"both to remind other people [about common errors] and to pat people on the back. Sometimes they do tremendous things," added the manager. (Pharmacy management team 79)

Thus, the classification and reporting of a medication error as an intervention set off organizational routines that simultaneously rewarded pharmacists both for their vigilant performance and for reporting it.

\section{Disincentives for reporting}

Medication errors reported to the hospital incident reporting system initiated a different series of organizational routines. The nursing unit supervisor ascertains whether or not the patient received the medication and was adversely affected, briefly investigates and describes the event, and submits the report to the medication incident reporting system. If the main responsibility for the incident is attributed to the pharmacy, then the incident report is relayed to the pharmacy for further investigation and analysis.

The hospital pharmacy sets in motion two parallel organizational routines-one for analyzing the incident and weighing the need for corrective action and the second for determining the accountability of the pharmacist involved. The pharmacy keeps track of the medication incidents attributed to each pharmacist, monitoring for frequency and for patterns of repeated errors. During the performance evaluation the individual pharmacist's record is compared with that of his colleagues.

Pharmacy staff members offered different interpretations of these pharmacy routines for maintaining accountability. Although the pharmacy promoted and implemented a nonpunitive policy toward those involved in medication incidents, the pharmacy staff differed in their perceptions of the negative consequences of being involved in a reportable incident, as an experienced pharmacist revealed:

"I do know that some pharmacists, particularly new pharmacists, are a little reluctant to be involved at all [in reportable incidents] because they think that they're in some way going to be penalized or reprimanded for it. I don't view it that way at all. I can only think that it would help the [patients], which is the whole point we are here." (Pharmacist 1)

Other pharmacy staff perceived that there were negative repercussions for being involved in a reportable medication incident because it "counted against them" in their performance evaluation, as a pharmacy technician explained:

"Yes, you're talked [to] about it [medication error] or perhaps reprimanded when it happens. And, yeah, when it is time for dollars and cents to come around, you'll see how much money you're going to get. Your performance is directly related to your increase in pay." (Pharmacy technician 18)

Pharmacists were in a position to notice medication errors that could be classified as reportable incidents, but they rarely reported them to the hospital. They not only detected mistakes in ordering and dispensing medications, but also after the drug was administered. For example, when updating a new dosage on the patient's computerized record, a pharmacist may discover an error that had previously gone unnoticed. However, despite their ability to identify these reportable errors, many pharmacists told us-and their managers confirmed-that the pharmacists never or rarely filed incident reports involving themselves or their colleagues.

As these examples illustrate, the classification of a medication error as an intervention versus a reportable incident can set in motion organizational routines that respectively enhance or detract from the performance evaluation of a pharmacist. These routines for distributing (or reducing) rewards provide pharmacists with incentives (or disincentives) for reporting medication errors.

\section{Definitions and data analysis}

The hospital maintained two separate databases: one for incidents (including medication errors discovered outside the pharmacy and reported to the hospital wide incident reporting system) and a second for interventions (including a subset of errors discovered within the pharmacy). The classification of medication errors and their compilation into two distinct databases has important implications for data 
analysis and learning. The maintenance of separate databases influenced who analyzed the data, the purpose for the data analysis, and who received the analysis results.

\section{Analyzing data from medication incident reports}

Medication incident reports from both pharmacy and nursing staff were combined in one hospital wide medication incident database. The pharmacy was given responsibility for the compilation and final analysis of all the medication related incidents. Highly skilled and experienced pharmacy staff members reviewed all of the medication incident reports for trends and to identify critical incidents that revealed system based problems that could recur in the pharmacy and the hospital. Based on these analyses of medication incident reports, the pharmacy occasionally changed its policies and procedures. The pharmacy also summarized the medication incident data and periodically presented it for review to the hospital Pharmacy and Therapeutics Committee that had the authority to make hospital wide changes in procedures, if required.

\section{Analyzing data from intervention reports}

The pharmacy analyzed intervention data mainly for internal purposes. The intervention data were used to identify topics that required further education and training for the pharmacy staff, according to a pharmacy manager. Although descriptive summaries of the frequency distributions of various types of interventions were sent to key decision makers in the pharmacy and the hospital, such as the Pharmacy and Therapeutics Committee, the data usually stayed within the purview of the pharmacy.

"There's also the pharmacy's system of pharmacists' interventions and that doesn't necessarily get looked at hospital-wide," as one high level hospital administrator explained (Administrator 67)

This resulted in decreased learning opportunities for physicians, especially residents, and for the hospital.

\section{Implications for residents learning}

Each intervention usually involved an interaction between a pharmacist and a physician in which the pharmacist called for a clarification of a prescription. When the pharmacist caught a prescription mistake such as a wrong dose or misplaced decimal point, it was defined as a pharmacist intervention rather than a physician error. To paraphrase one pharmacist: Physicians do not make errors, they make corrections. Another said:

"Because we don't want to offend, we don't want to say that, admit that physicians do make mistakes". (Pharmacist 15)

Thus, prescribing errors were classified as pharmacist interventions and physician corrections, rather than physician mistakes.

The classification of prescribing errors as interventions focused attention on the pharmacists' contribution to the hospital, but it distracted attention from the physicians. Accordingly, intervention reporting triggered organizational routines for analyzing data for pharmacists, but not for physicians. The interventions were tallied for each pharmacist, as noted earlier, but they were not computed for the residents or for the group of physicians on each medical service, noted a pharmacist.

"They [pharmacists] would say: 'Call Dr so and so...to change a dose for so and so'. But a physician would not be written up for a mistake. You hope that when you call him with the intervention that he learns from it." (Pharmacist 15)

An individual resident may learn from a particular prescribing error, but intervention data including information on patterns in prescribing errors was not relayed directly to the residents or those who train them. A member of the pharmacy management team remarked:

"...but we're not sharing it [intervention data] the way we should, I don't think. It's good information but we're not doing anything outside of us fixing things." (Pharmacy management team 76)

An attending physician directly involved in resident training exclaimed:

"I honestly had no idea that they [the pharmacy] were trying to do so much work in terms of quantitating (sic) their errors. I had no idea that was going on. I would have loved to have that information to feed back to my guys [residents] to make sure that we're not making more mistakes...". (Physician 66)

The intervention data analysis therefore did not enable residents to learn from the experience of others and could not inform the modification of resident training routines. The classification of prescribing errors in terms of pharmacist performance rather than physicians learning from their mistakes illustrates how the classification of medication errors can influence their analysis.

\section{Implications for hospital learning}

At the hospital level it was difficult to assess the patterns in medication errors because errors that occurred at different stages of the process (for example, prescribing and dispensing) tended to be sorted into two different databases. As a pharmacy management team member explained:

"The majority of the interventions reflect prescribing errors, which could be included in our other system; but we choose not to, because ... to meld them together would not serve our purpose." (Pharmacy management team 79)

The intervention database focused on prescribing errors caught within the pharmacy, whereas errors in the drug dispensing and administration were reported to the medication incident database. Maintaining separate databases for reportable incidents and interventions therefore divided the data from otherwise interdependent components of the medication process.

From our interviews we also learned that errors resulting from similar system problems in the medication ordering process could be found in both the intervention and incident database. As a key pharmacy administrator explained:

"The only difference between the 1500 interventions and the 5 prescribing errors that got through in a quarter is that they got through [to the patient]. There's no difference. So, the dose was wrong here, and the dose was wrong here 1500 times; well ... it's the same underlying problem." (Pharmacy management team 79)

For example, if a physician wrote an incorrect prescription based on an outdated and inaccurate standardized order form, it could be classified and reported as an intervention or, alternatively, as an incident due solely to chance, depending on when and where the error was first noticed. The use of an outdated standardized prescription form may have been disregarded as an outlier if only a few cases were recorded in the incident database and a few in the separate intervention database. The emerging trend would have become apparent if all the reports had been pooled and analyzed in a unified database. As this example suggests, if similar events are reported to and stored in separate databases, such as those for interventions and incidents, it may obstruct the view of trends emerging from infrequent but important events.

\section{DISCUSSION}

We propose that the definition and categorization of safety related events influences key organizational routines. Specifically, the classification of an event into one category or another can influence the activation of organizational routines for gathering information, allocating incentives, and analyzing data (box 1). The choice of how to define a safety 
Box 1 Research propositions

\section{Definitions and information collection}

\section{Proposition 1}

Under-reporting can result from how a hospital-as an organization or units within it-defines and classifies medication errors and other safety related events.

\section{Definitions and incentive distribution}

Proposition 2

The classification of a medication error into different categories influences the perceived incentives and disincentives for incident reporting.

\section{Definitions and data analysis}

Proposition 3

The classification of events into alternative categories can influence the choice of organizational routines for data analysis and learning.

\section{Proposition 4}

If similar safety related events are reported to and stored in separate databases (divided by professional or organizational accountability), it will obstruct the view of trends emerging from infrequent but important events.

Definitions, incentive distribution, and information gathering

Proposition 5

Event classification and incentive distribution processes that hinder learning at the hospital level may promote information gathering and learning within hospital departments and vice versa.

related event and how to apply the definition in practice can therefore have critical implications for a hospital's ability to gather and analyze information gleaned from a patient safety reporting system.

\section{Definitions and information collection: under- reporting incidents}

By focusing on how safety related events are defined in practice, we found that some medication errors were not reported to the hospital because, depending on where the event was detected, they did not fit the working definition of a reportable incident. Consequently, hospital decision makers may remain unaware of potentially harmful medication errors.

We found an overlooked source of the ubiquitous ${ }^{14} 15$ under-reporting to hospital incident reporting systems. The reluctance to file incident reports has been attributed to many factors including shared perceptions of team members, ${ }^{16}$ fear of punishment, and lack of time by busy healthcare professionals. ${ }^{17}$ Our findings suggest that underreporting occurred because some of the potential medication incidents were "defined away"-meaning that, because the event did not meet the pharmacy's working definition of an error, it was not considered a reportable event. Similar phenomena have been observed in an ethnographic study of hospital nurses ${ }^{18}$ and in research on aviation safety information systems.

We speculate that physicians also define away medication errors. For example, if a physician intervenes and reverses the effects of a mistaken dosage, he/she may no longer consider it a problem and therefore not classify it as a reportable error. Physicians, like other professionals, ${ }^{19}$ may not report mishaps because they classify them as their expert "solutions" rather than as "problems" generated by the workings of the hospital system. Hence, whereas previous research has focused mainly on the decision whether or not to report a medical error, we propose that under-reporting can also result from how the hospital-as an organization or units within it—defines and classifies events.

\section{Definitions and incentive distribution}

The results of this study illustrate how the classification of medication errors activates alternative routines for allocating incentives for reporting. Specifically, the classification of similar medication errors as an intervention or a reportable incident could set in motion organizational routines that respectively enhanced or detracted from the performance evaluation of a pharmacist. These routines for distributing (or reducing) rewards provided pharmacists with incentives (or disincentives) for reporting medication errors. We hypothesize that the pharmacy management's positive response to staff who made and reported interventions may have increased intervention reporting. An upsurge in intervention reporting, in turn, may have expanded the pharmacy's capacity to learn by making available a larger pool of information for analysis and learning. In contrast, although the pharmacy maintained a non-punitive policy towards those involved in medication incidents, the pharmacy staff differed in their perceptions of the negative consequences of being involved in such incidents. Indeed, pharmacists rarely filed incident reports involving themselves or their colleagues. We therefore conclude that the classification of a medication error into one category or another can influence the perceived incentives and disincentives for incident reporting.

\section{Definitions and data analysis}

If prescription errors were detected before drugs were dispensed by the pharmacy-including errors attributed to physicians - they were classified, reported, and compiled as pharmacist interventions rather than as reportable incidents. By maintaining separate databases for incidents and interventions, the hospital truncated the databases it used for analyzing patterns in medication errors. The capacity of the hospital to analyze and learn from its experience may therefore have been limited. ${ }^{8}$

Firstly, the data on medication incidents systematically underestimated the number of errors that occurred during the prescribing of medications compared with those that occurred during drug dispensing and administration. This was because physician prescribing errors were classified and reported as pharmacist interventions rather than as reportable incidents, and then compiled in two separate databases.

Secondly, whereas the hospital pharmacy learned from medication errors, changing its procedures based on previous mistakes, the learning activities of the hospital may have been hindered by the lack of an integrated database for monitoring medication errors. Specifically, we hypothesize that it limited the efforts of decision makers to identify precursors to adverse drug events because of the difficulties of monitoring trends among similar medication errors that were divided between two different databases. We therefore suggest that the classification of events into alternative categories can influence the choice of organizational routines for data analysis and learning.

\section{Definitions, incentive distribution, and information gathering}

We observed that particular organizational processes that hindered "global" data gathering also fostered "local" learning. Specifically, some of the routines for event classification, incentive distribution, and information gathering within the pharmacy promoted organizational learning 
Box 2 Key questions for hospital administrators and health service researchers

For hospital administrators and health services researchers, we propose the following key questions:

- What are the formal and informal definitions of safety related events? How are they applied in practice?

- How does the definition of events channel managers' attention?

- How does the classification of events into one category or another trigger organizational routines for gathering and analyzing information?

- Does the definition of an event trigger the allocation of incentives or disincentives? Are the punishments or rewards consistent with the underlying purpose of the categorization scheme?

within the department but inadvertently diverted information from the hospital-for example, the labeling of errors detected in the pharmacy as "non-events" directly reduced the flow of medication error data to the hospital. Concurrently, by redefining these errors in positive terms such as "interventions" and "good catches", the pharmacy fostered internal conditions conducive to information gathering and learning. Pharmacy routines further bolstered efforts to gather information by rewarding pharmacists who engaged in and reported interventions. This illustrates how learning processes can operate differently at higher and lower levels, or in various subunits of the organization, or in what has been described as an "ecology of learning". ${ }^{20}$ We therefore postulate that the confluence of organizational processes such as event classification and incentive allocation that hinder learning at the hospital level may promote information gathering and learning within the pharmacy department or within patient care units, and vice versa.

\section{Study limitations}

We have attempted to highlight organizational processes that will stimulate discussion among hospital administrators and generate hypotheses for further research, but we recognize that the study has some limitations. Firstly, the findings are based on the preliminary analysis of interview data, providing

\section{Key messages}

- The choice of event definitions and classification schemes for hospital incident reporting systems influences the ability of a hospital to learn from medical errors.

- Hospital administrators and researchers should consider asking the following questions about incident reporting in their organizations:

- What are the informal and formal definitions of safety related events?

- How do definitions channel the attention of providers and managers?

- How does event classification influence data gathering and analysis?

- Does the distribution of rewards and accountability measures provide incentives (or disincentives) for event classification and reporting? examples from interviews rather than a systematic analysis of all the data. Secondly, the results may not be generally applicable because the findings may be unique to the one hospital we studied or they may be limited in scope to urban tertiary care teaching hospitals. Thirdly, the study focused on classifying events as incidents or interventions, one of many types of classification. In future studies we will explore classification by professional accountability (for example, pharmacists, nurses, or physicians) and by event outcome (for example, near miss and adverse event). Fourthly, the choice of examples and their interpretation may have been biased because the first author conducted both the aviation and hospital studies. Finally, we hypothesized that event categorization results in different ways of analyzing and learning from the events. However, it may be that the categorization schemes reflect fundamental differences in the events, so it may be appropriate for the events to be analyzed in different ways.

\section{Posing questions for research and practice}

Examples from our research are used to illustrate our ideas and to generate research questions rather than to draw generalizable conclusions. Yet, the in depth study of one hospital raises important questions to guide future research and offer new perspectives on event reporting. The key questions for hospital administrators and health service researchers to consider for future studies to improve patient safety are shown in box 2 .

Thoughtful consideration of these key questions by researchers, hospital administrators, and healthcare providers may lead to a greater understanding of how errors are defined, classified, and analyzed within healthcare organizations. By understanding how we classify and label our experience, we hope to improve our capacity to learn from it and, ultimately, to improve patient safety.

\section{ACKNOWLEDGEMENTS}

The authors are grateful to the Aetna Foundation's Quality Care Research Fund for funding this research.

\section{Authors' affiliations}

M Tamuz, University of Tennessee Health Science Center, Center for Health Services Research, Memphis, TN 38163, USA

E J Thomas, The University of Texas Health Science Center at Houston, Medical School, Department of Medicine and Division of General Internal Medicine, Houston, Texas 77030, USA

K E Franchois, The University of Texas Health Science Center at Houston, School of Public Health, Houston, Texas 77030, USA

\section{REFERENCES}

1 Hofer TP, Kerr EA, Hayward RA. What is an error? Eff Clin Pract 2000;3:261-9.

2 Reason J. Human error. Cambridge, UK: Cambridge University Press, 1990.

3 Rosch E. Cognitive reference points. Cognitive Psychol 1975;7:532-47.

4 Rosch E. Principles of categorization. In: Rosch E, Lloyd B, eds. Cognition and categorization. Hillsdale, NJ: Lawrence Erlbaum, 1978:27-47.

5 Dutton J, Jackson S. Categorizing strategic issues: links to organizational action. Acad Manage Rev 1987;12:76-90.

6 March JG, Simon HA. Organizations, 2nd ed. Cambridge, MA: Blackwell, 1993 (originally published in 1958 by Wiley).

7 Tamuz M. Defining away dangers: a study in the influences of managerial cognition on information systems. In: Lant TK, Shapira Z, eds. Organizational cognition: computation and interpretation. Mahwah, NJ: Lawrence Erlbaum, 2000:157-83.

8 Tamuz M. Learning disabilities for regulators: the perils of organizational learning in the air transportation industry. Admin Soc 2001;33:276-302.

9 Thomas EJ, Helmreich RL. Will airline safety models work in medicine? In: Sutcliffe KM, Rosenthal MM, eds. Medical error. San Francisco: Jossey-Bass, 2002:217-34.

10 Barach PS, Small SD. Reporting and preventing medical mishaps: lessons from non-medical near miss reporting systems. BMJ 2000;320:759-63.

11 Billings CE. Some hopes and concerns regarding medical event-reporting systems: lessons from the NASA safety reporting system. Arch Pathol Lab Med 1998; 122:214-5. 
12 Thomas EJ, Studdert DM, Burstin HR, et al. Incidence and types of adverse events and negligent care in Utah and Colorado in 1992. Med Care 2000;38:261-71.

13 Miles MB, Huberman AM. Qualitative data analysis: an expanded sourcebook. Thousand Oaks, CA: Sage Publications, 1994.

14 Kohn LT, Corrigan JM, Donaldson MS, eds. To err is human: building a safer health system. Institute of Medicine. Washington, DC: National Academy Press, 1999.

15 Cullen DJ, Bates DW, Small SD, et al. The incident reporting system does not detect adverse drug events: a problem for quality improvement. Jt Comm J Qual Improv 1995;21:541-8.
16 Edmonson AC. Learning from mistakes is easier said than done: group and organizational influences on the detection and correction of human error. J Appl Behav Sci 1996;32:5-28.

17 Vincent C, Stanhope N, Crowley-Murphy M. Reasons for not reporting adverse incidents: an empirical study. J Eval Clin Pract 1999:5:13-21.

18 Baker HM. Rules outside the rules for administration of medication: a study in New South Wales, Australia. Image J Nurs Sch 1997;29:155-8.

19 Levinthal D, March JG. The myopia of learning. Strat Manage J 1993; 14:95-112.

20 Levitt B, March JG. Organizational learning. Annu Rev Soc $1988 ; 14: 319-40$

\section{Clinical Evidence-Call for contributors}

Clinical Evidence is a regularly updated evidence based journal available worldwide both as a paper version and on the internet. Clinical Evidence needs to recruit a number of new contributors. Contributors are health care professionals or epidemiologists with experience in evidence based medicine and the ability to write in a concise and structured way.

\section{Currently, we are interested in finding contributors with an interest in} the following clinical areas:

Altitude sickness; Autism; Basal cell carcinoma; Breast feeding; Carbon monoxide poisoning; Cervical cancer; Cystic fibrosis; Ectopic pregnancy; Grief/bereavement; Halitosis; Hodgkins disease; Infectious mononucleosis (glandular fever); Kidney stones; Malignant melanoma (metastatic); Mesothelioma; Myeloma; Ovarian cyst; Pancreatitis (acute); Pancreatitis (chronic); Polymyalgia rheumatica; Post-partum haemorrhage; Pulmonary embolism; Recurrent miscarriage; Repetitive strain injury; Scoliosis; Seasonal affective disorder; Squint; Systemic lupus erythematosus; Testicular cancer; Varicocele; Viral meningitis; Vitiligo

However, we are always looking for others, so do not let this list discourage you.

\section{Being a contributor involves:}

- Appraising the results of literature searches (performed by our Information Specialists) to identify high quality evidence for inclusion in the journal.

- Writing to a highly structured template (about 2000-3000 words), using evidence from selected studies, within 6-8 weeks of receiving the literature search results.

- Working with Clinical Evidence Editors to ensure that the text meets rigorous epidemiological and style standards.

- Updating the text every eight months to incorporate new evidence.

- Expanding the topic to include new questions once every 12-18 months.

If you would like to become a contributor for Clinical Evidence or require more information about what this involves please send your contact details and a copy of your CV, clearly stating the clinical area you are interested in, to Claire Folkes (cfolkes@bmigroup.com).

\section{Call for peer reviewers}

Clinical Evidence also needs to recruit a number of new peer reviewers specifically with an interest in the clinical areas stated above, and also others related to general practice. Peer reviewers are health care professionals or epidemiologists with experience in evidence based medicine. As a peer reviewer you would be asked for your views on the clinical relevance, validity, and accessibility of specific topics within the journal, and their usefulness to the intended audience (international generalists and health care professionals, possibly with limited statistical knowledge). Topics are usually 2000-3000 words in length and we would ask you to review between 2-5 topics per year. The peer review process takes place throughout the year, and our turnaround time for each review is ideally 10-14 days.

If you are interested in becoming a peer reviewer for Clinical Evidence, please complete the peer review questionnaire at www.clinicalevidence.com or contact Claire Folkes(cfolkes@bmigroup.com). 